\title{
Endotracheal intubation using a fiberoptic bronchoscope and laryngeal mask airway in ICU
}

\author{
Eun Yong Chung, Yee-Suk Kim, Joo-Hyun Yoo, and In-Soo Han \\ Department of Anesthesiology and Pain Medicine, The Catholic University of Korea, Bucheon St. Mary's Hospital, Bucheon, Korea
}

Endotracheal intubation for patients undergoing spine surgery in intensive care unit (ICU) needs a careful attention as it can be associated with unexpected difficulties, and, if necessary, the use of flexible fiberoptic bronchoscope (FFB) or laryngeal mask airway (LMA) is considered [1]. In particular, LMA can be a measure for a successful airway management before percutaneous tracheostomy in cases of difficult endotracheal intubation [2]. A combitube combining endotracheal tube (ETT) and esophageal obturator is also an excellent tool to be able to prevent the risk of aspiration and these tools can be utilized easily by even surgeons not familiar with airway control [3]. However, it is hard to put these tools in the case of airway management for a long time because of the risk of esophageal reflux or the aspiration of oral fluid and the instability of patients. Against this background, the authors performed endotracheal intubation successfully through FFB with LMA as a passage after securing airway through LMA in a patient treated with spine surgery for which endotracheal intubation was expected to be difficult to need a long-term airway management and the case are reported along with literature review.

A 66-year old man whose body weight and height were $73 \mathrm{~kg}$ and $158 \mathrm{~cm}$, respectively, was diagnosed as ossification of 3, 4 cervical spine posterior longitudinal ligament and was treated with microdiscectomy, removal of vertebral body and anterior cervical spinal fusion. There was no intraoperative abnormal finding or sign. As spontaneous breathing was sufficient, he was transferred to the neurosurgery ICU after extubation immediately after the surgery by following the request of the surgeon. Although around $2 \mathrm{~mm}$ edema was observed to postoperative (PO) $5^{\text {th }}$ day after the transfer of ICU, it was not a level to provoke any problem. On PO $6^{\text {th }}$ day, the sudden increase of the edema on the operation site and anxiety of the patient led to dyspnea, and endotracheal intubation was tried by the surgeon. But, the intubation could not be conducted because of the severe edema, the limited movement of the operation site and the lack of cooperation of the patient, so we were asked to deal with the case. The possibility of massive hemorrhage on the operation site was excluded.

Although there was no abnormal finding in vital signs and consciousness, he complained of anxiety along with severe dyspnea. Endotracheal intubation was tried after intravenous administration of $2 \mathrm{mg}$ midazolam, but the neck was not extended and the intraoral structure could not be checked due to severe edema. As the changed location of airway caused by the edema was suspected, it was considered that the examination on the accurate intraoral structure and the minimal stimulus and neck movement were necessary. Endotracheal intubation using disposable LMA and FFB was tried (Fig. 1). In our hospital, Intubating LMA (LMA Fastrach) was exhausted so that disposable LMA was used in this study.

In 50 seconds after the administration of $50 \mathrm{mg}$ Succinylcholine, LMA was inserted four times. Appropriate ventilation was checked and then I.D.6.5 mm ETT was put to FFB and the airway was investigated through the FFB. ETT was inserted into the trachea with FFB as an introducer and FFB and then LMA were removed for a successful endotracheal intubation. After that time, proper ventilation was found to succeed in the airway management. On PO $12^{\text {th }}$ day, ETT was removed.

Special methods for endotracheal intubation should be

Corresponding author: In-Soo Han, M.D., Department of Anesthesiology and Pain Medicine, The Catholic University of Korea, Bucheon St. Mary's Hospital, 2, Sosa-dong, Wonmi-gu, Bucheon 420-717, Korea. Tel: 82-32-340-2158, Fax: 82-32-340-2545, E-mail: orangel0@nate.com (c) This is an open-access article distributed under the terms of the Creative Commons Attribution Non-Commercial License (http:// creativecommons.org/licenses/by-nc/3.0/), which permits unrestricted non-commercial use, distribution, and reproduction in any medium, provided the original work is properly cited. 


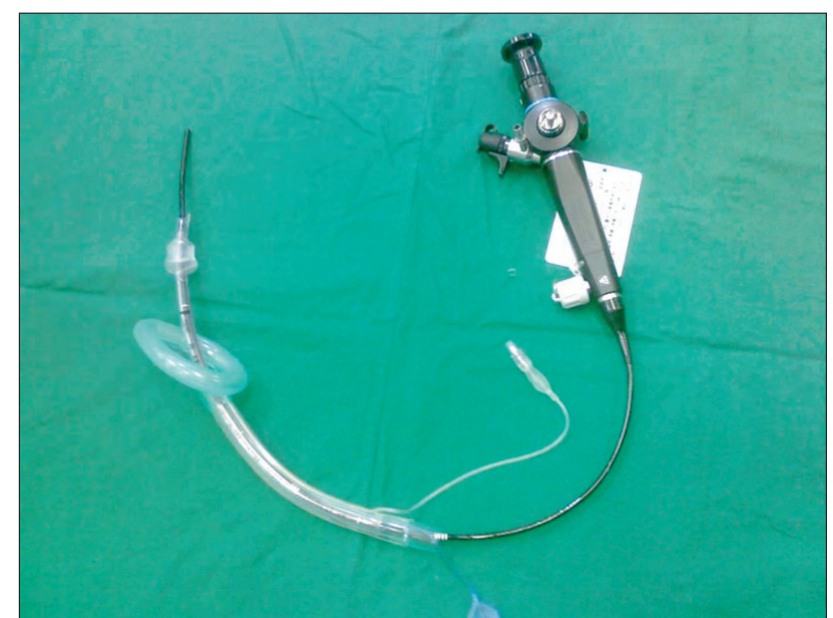

Fig. 1. Fiberoptic bronchoscope inducing endotracheal tube has been inserted within the LMA.

devised for patients who are suspected to have cervical spine injury or are treated with head and neck surgery in ICU. Majernick et al. [4] reported that the movement of the cervical spine was observed during endotracheal intubation in a patient with Philadelphia collar, and any measure could not suppress the movement.

The use of FFB needs very experienced skills and it has difficulties such as the performance of nerve block to prevent laryngopharyngeal reflex. Jun et al. [5] said that successful endotracheal intubation was conducted rapidly without discomfort of patients by using LMA and FFB in difficult cases. In particular, for the method of Jun et al. [5] utilizing LMA and FFB, even surgeons not familiar with FFB can use it easily and the intubation can be performed within a short time and the risk following the delay of airway management is not associated because the airway is already secured. In particular, the cervical spine does not move in patients with cervical spine injury.

In conclusion, various methods for endotracheal intubation have been tried in patients for whom endotracheal intubation is expected to be hard in ICU. When spinal cord injury is suspected in patients with cervical spine injury or when the anatomical structure of the airway is suspected to change due to severe edema after head and neck surgery, emergency tracheostomy has been performed if endotracheal intubation fails. However, this endotracheal intubation using FFB with LMA as an introducer is considered to be a fast, safe and comfortable intubation.

\section{References}

1. Schoenhage KO, Koenig HM. Unanticipated difficult endotracheal intubations in patients with cervical spine instrumentation. Anesth Analg 2006; 102: 960-3.

2. Divatia JV, Kulkarni AP, Sindhkar S, Upadhye SM. Failed intubation in the intensive care unit managed with laryngeal mask airway and percutaneous tracheostomy. Anaesth Intensive Care 1999; 27: 40911.

3. Yardy N, Hancox D, Strang T. A comparison of two airway aids for emergency use by unskilled personnel: the Combitube and laryngeal mask. Anaesthesia 1999; 54: 181-3.

4. Majernick TG, Bieniek R, Houston JB, Hughes HG. Cervical spine movement during orotracheal intubation. Ann Emerg Med 1986; 15: 417-20.

5. Jun JH, Seung IS, Cho SY, Suh JK. Endotracheal intubation with laryngeal mask airway and fiberoptic bronchoscope. Korean J Anesthesiol 1993; 26: 1029-34. 